Journal of Applied AnALysis

Vol. 9, No. 2 (2003), pp. 275-286

\title{
ON MARCZEWSKI-BURSTIN REPRESENTATIONS OF ALGEBRAS AND IDEALS
}

\author{
M. BALCERZAK and J. RZEPECKA
}

Received November 11, 2001 and, in revised form, September 2, 2003

\begin{abstract}
We study MB-representations of algebras and ideals when they are relativized to a subset, and when one considers the operations of sum and intersection for families of algebras and ideals. We observe that the algebras $\Delta_{\alpha}^{0}, 3 \leq \alpha<\omega_{1}$, on $\mathbb{R}$ are MB-representable under $\mathrm{GCH}$. We find a class of topological spaces in which the algebra of clopen sets is MB-representable.
\end{abstract}

\section{Introduction}

Our notation is standard. (See [7].) Let $X \neq \emptyset$. Define two operations $S_{X}, S_{X}^{0}: \mathcal{P}(\mathcal{P}(X) \backslash\{\emptyset\}) \rightarrow \mathcal{P}(\mathcal{P}(X))$ given by

$S_{X}(\mathcal{F})=\{E \subset X:(\forall A \in \mathcal{F})(\exists B \in \mathcal{F}) B \subset A \cap E \vee B \subset A \backslash E\}$,

$S_{X}^{0}(\mathcal{F})=\{E \subset X:(\forall A \in \mathcal{F})(\exists B \in \mathcal{F}) B \subset A \backslash E\}$.

In [2] (see also [19]) it was observed that $S_{X}(\mathcal{F})$ forms an algebra of sets and $S_{X}^{0}(\mathcal{F})$ forms an ideal of sets. Obviously $S_{X}^{0}(\mathcal{F}) \subset S_{X}(\mathcal{F})$ and $X \in S_{X}(\mathcal{F})$. Throughout the paper we assume that an algebra of subsets of $X$ contains

2000 Mathematics Subject Classification. 54H05, 06E25, 03E15.

Key words and phrases. Marczewski operation, sums of algebras of sets, ambiguous Borel sets, clopen sets.

ISSN 1425-6908（C) Heldermann Verlag. 
the set $X$. Operations $S_{X}$ and $S_{X}^{0}$ were considered by Marczewski [22], and earlier by Burstin [6]. (See also [17].) The authors of [5], [2] and [1] proved that several algebras and ideals of subsets of $X$ are of the form $S_{X}(\mathcal{F})$ and $S_{X}^{0}(\mathcal{F})$. This problem was also investigated for pairs $(\Sigma, \mathcal{I})$ where $\mathcal{I}$ is an ideal in an algebra $\Sigma$. If the respective representation exists, we say that $\Sigma$ (or $(\Sigma, \mathcal{I})$ ) is Marczewski-Burstin representable or briefly, MB-representable. Some questions on this topic remain open, for instance it is still not known whether it can be proved in ZFC that the algebra of Borel subsets of $\mathbb{R}$ is MB-representable. (See [1].) In our paper we continue studies connected with MB-representations. If $X$ is fixed, we write $S$ and $S^{0}$ instead of $S_{X}$ and $S_{X}^{0}$.

\section{Relativization, sums and intersections}

Let $\Sigma$ be an algebra of subsets of $X$ and let $\emptyset \neq Y \in \Sigma$. Then the family $\Sigma_{Y}=\{A \cap Y: A \in \Sigma\}$ forms an algebra of subsets of $Y$. This is a natural relativization of the algebra $\Sigma$ to subsets of $Y$. Also, if $\mathcal{I} \subset \Sigma$ is an ideal of sets and $Y \notin \mathcal{I}$ then $\mathcal{I}_{Y}=\{A \cap Y: A \in \mathcal{I}\}$ forms an ideal of subsets of $Y$.

Theorem 1. Assume that $\mathcal{F} \subset \mathcal{P}(X) \backslash\{\emptyset\}, \Sigma=S_{X}(\mathcal{F}), \mathcal{I}=S_{X}^{0}(\mathcal{F})$ and $Y \in \Sigma \backslash \mathcal{I}$. Then $\Sigma_{Y}=S_{Y}\left(\mathcal{F}_{Y}\right)$ and $\mathcal{I}_{Y}=S_{Y}^{0}\left(\mathcal{F}_{Y}\right)$ where $\mathcal{F}_{Y}=\mathcal{F} \cap \mathcal{P}(Y)$.

Proof. Since $Y \in \Sigma$, we have $\Sigma_{Y}=\Sigma \cap \mathcal{P}(Y)$ and $\mathcal{I}_{Y}=\mathcal{I} \cap \mathcal{P}(Y)$.

We will show that $\Sigma_{Y} \subset S_{Y}\left(\mathcal{F}_{Y}\right)$. Let $E \in \Sigma_{Y}$, thus $E \in \Sigma$ and $E \subset Y$. Let $A \in \mathcal{F}_{Y}$, thus $A \in \mathcal{F}$ and $A \subset Y$. Since $E \in \Sigma=S_{X}(\mathcal{F})$, there is a $C \in \mathcal{F}$ such that either $C \subset A \cap E$ or $C \subset A \backslash E$. Obviously $C \in \mathcal{F}_{Y}$. Hence $E \in S_{Y}\left(\mathcal{F}_{Y}\right)$.

We will show that $S_{Y}\left(\mathcal{F}_{Y}\right) \subset \Sigma_{Y}$. Let $E \in S_{Y}\left(\mathcal{F}_{Y}\right)$. It is enough to prove that $E \in S_{X}(\mathcal{F})$. Let $A \in \mathcal{F}$. If there is a $B \in \mathcal{F}$ such that $B \subset A \cap Y$, then $B \in \mathcal{F}_{Y}$ and from $E \in S_{Y}\left(\mathcal{F}_{Y}\right)$ it follows that there is a $C \in \mathcal{F}_{Y}$ such that either $C \subset B \cap E$ or $C \subset B \backslash E$. Since $C \in \mathcal{F}_{Y}$, we have $C \in \mathcal{F}$. If there is no $B \in \mathcal{F}$ such that $B \subset A \cap Y$ then, since $Y \in \Sigma=S_{X}(\mathcal{F})$, there is a $C \in \mathcal{F}$ for which $C \subset A \backslash Y$. Hence $C \subset A \backslash E$. So $E \in S_{X}(\mathcal{F})$.

Analogously, one can show that $\mathcal{I}_{Y}=S_{Y}^{0}\left(\mathcal{F}_{Y}\right)$.

Now, let us consider a kind of the inverse problem. Let $T \neq \emptyset$. For each $t \in T$, let $Y_{t}$ be a nonempty subset of $X$. Assume that for each $t \in T$, an algebra $\Sigma^{t}$ and an ideal $\mathcal{I}^{t}$ of subsets of $Y_{t}$ are given. It is easy to check that the families

$$
\Sigma=\bigcap_{t \in T}\left\{E \subset X: E \cap Y_{t} \in \Sigma^{t}\right\} \text { and } \mathcal{I}=\bigcap_{t \in T}\left\{E \subset X: E \cap Y_{t} \in \mathcal{I}^{t}\right\}
$$


form an algebra and an ideal of subsets of $X$, respectively. We denote $\Sigma=\bigoplus_{t \in T} \Sigma^{t}$ and $\mathcal{I}=\bigoplus_{t \in T} \mathcal{I}^{t}$. Observe that if any two distinct members of $\mathcal{Y}=\left\{Y_{t}: t \in T\right\}$ are disjoint, we have $\Sigma_{Y_{t}}=\Sigma^{t}$ and $\mathcal{I}_{Y_{t}}=\mathcal{I}^{t}$ for each $t \in T$. The operation $\bigoplus$ is analogous to the sum of topological spaces [9], however, in general we do not require the disjointness of sets $Y_{t}, t \in T$.

Theorem 2. Assume that $T \neq \emptyset$ and let $Y_{t} \in \mathcal{P}(X) \backslash\{\emptyset\}, \mathcal{F}_{t} \subset \mathcal{P}\left(Y_{t}\right) \backslash\{\emptyset\}$ be given for each $t \in T$. Assume additionally, for any $t_{1}, t_{2} \in T$, the following condition

$$
\left(\forall A_{1} \in \mathcal{F}_{t_{1}}, \forall A_{2} \in \mathcal{F}_{t_{2}}\right) \quad A_{1} \subset A_{2} \Rightarrow A_{1} \in \mathcal{F}_{t_{2}} .
$$

If $\Sigma^{t}=S_{Y_{t}}\left(\mathcal{F}_{t}\right)$ and $\mathcal{I}^{t}=S_{Y_{t}}^{0}\left(\mathcal{F}_{t}\right)$ then, for $\Sigma=\bigoplus_{t \in T} \Sigma^{t}$ and $\mathcal{I}=\bigoplus_{t \in T} \mathcal{I}^{t}$, we have $\Sigma=S_{X}(\mathcal{F})$ and $\mathcal{I}=S_{X}^{0}(\mathcal{F})$ where $\mathcal{F}=\bigcup_{t \in T} \mathcal{F}_{t}$.

Proof. To show $\Sigma \subset S_{X}(\mathcal{F})$, consider an $E \in \Sigma$ and an $A \in \mathcal{F}$. Hence $A \in \mathcal{F}_{t}$ for some $t \in T$. Clearly $E \cap Y_{t} \in \Sigma^{t}=S_{Y_{t}}\left(\mathcal{F}_{t}\right)$. Thus there exists a $B \in \mathcal{F}_{t}$ such that either $B \subset\left(A \cap Y_{t}\right) \cap\left(E \cap Y_{t}\right)$ or $B \subset\left(A \cap Y_{t}\right) \backslash\left(E \cap Y_{t}\right)$. Consequently, $B \in \mathcal{F}$ and either $B \subset A \cap E$ or $B \subset A \backslash E$. Hence $E \in S_{X}(\mathcal{F})$. (In this part of proof we do not use $(\star)$.)

To show $S_{X}(\mathcal{F}) \subset \Sigma$, consider an $E \in S_{X}(\mathcal{F})$ and a $t \in T$. We want to prove that $E \cap Y_{t} \in S_{Y_{t}}\left(\mathcal{F}_{t}\right)$. Let $A \in \mathcal{F}_{t}$. Obviously, $A \in \mathcal{F}$. Since $E \in S_{X}(\mathcal{F})$, there is a $B \in \mathcal{F}$ such that either $B \subset A \cap E$ or $B \subset A \backslash E$. By the definition of $\mathcal{F}$ there is a $t_{1} \in T$ such that $B \in \mathcal{F}_{t_{1}}$. Since $B \subset A$, we have $B \in \mathcal{F}_{t}$, by $(\star)$. Thus, either $B \subset A \cap\left(E \cap Y_{t}\right)$ or $B \subset A \backslash\left(E \cap Y_{t}\right)$. Hence $E \cap Y_{t} \in S_{Y_{t}}\left(\mathcal{F}_{t}\right)$.

The proof of $\mathcal{I}=S_{X}^{0}(\mathcal{F})$ is analogous.

Remark 1. A. Bartoszewicz has observed that $(\star)$ in Theorem 2 can be replaced by a weaker condition

$$
\left(\forall A_{1} \in \mathcal{F}_{t_{1}}, \forall A_{2} \in \mathcal{F}_{t_{2}}\right)\left(A_{1} \subset A_{2} \Rightarrow\left(\exists A \in \mathcal{F}_{t_{2}}\right) A \subset A_{1}\right) .
$$

The proof needs only minor modification. Note that condition $(\star)$ is fulfilled, if any two distinct sets $Y_{t_{1}}, Y_{t_{2}}$ are disjoint. This enables one to produce new examples of MB-representable algebras and ideals from the known examples.

It is obvious that the intersection of a family of algebras (ideals) is again an algebra (ideal). Is that intersection MB-representable, provided all the factors are MB-representable? From Theorem 2 we can infer the affirmative answer in some special case. 
Corollary 1. Let $T \neq \emptyset$ and let $\mathcal{F}_{t} \subset \mathcal{P}(X) \backslash\{\emptyset\}$ be given for each $t \in T$. Assume, for any $t_{1}, t_{2} \in T$, the following condition

$$
\left(\forall A_{1} \in \mathcal{F}_{t_{1}}, \forall A_{2} \in \mathcal{F}_{t_{2}}\right) \quad A_{1} \subset A_{2} \Rightarrow A_{1} \in \mathcal{F}_{t_{2}} .
$$

Then $\bigcap_{t \in T} S\left(\mathcal{F}_{t}\right)=S\left(\bigcup_{t \in T} \mathcal{F}_{t}\right)$ and $\bigcap_{t \in T} S^{0}\left(\mathcal{F}_{t}\right)=S^{0}\left(\bigcup_{t \in T} \mathcal{F}_{t}\right)$.

Proof. Put $Y_{t}=X$ for each $t \in T$, observe that $\bigoplus_{t \in T} S\left(\mathcal{F}_{t}\right)=\bigcap_{t \in T} S\left(\mathcal{F}_{t}\right)$, $\bigoplus_{t \in T} S^{0}\left(\mathcal{F}_{t}\right)=\bigcap_{t \in T} S^{0}\left(\mathcal{F}_{t}\right)$ and use Theorem 2 .

Now, we are going to show some situations where Corollary 1 applies. For an ideal $\mathcal{I} \subset \mathcal{P}(X)$ we denote

$$
\operatorname{add}(\mathcal{I})=\min \{|\mathcal{G}|: \mathcal{G} \subset \mathcal{I} \& \bigcup \mathcal{G} \notin \mathcal{I}\}
$$

We say that two ideals $\mathcal{I}, \mathcal{J} \subset \mathcal{P}(X)$ are orthogonal if there is a set $E \subset X$ such that $E \in \mathcal{I}$ and $X \backslash E \in \mathcal{J}$. We say that two families $\mathcal{F}, \mathcal{G} \subset \mathcal{P}(X) \backslash\{\emptyset\}$ are mutually coinitial (in symbols $\mathcal{F} \sim \mathcal{G}$ ), if

$$
(\forall A \in \mathcal{F})(\exists B \in \mathcal{G}) B \subset A \text { and }(\forall A \in \mathcal{G})(\exists B \in \mathcal{F}) B \subset A .
$$

(See [2].) It is easy to check that relation $\sim$ is transitive and that $\mathcal{F} \sim \mathcal{G}$ implies $S(\mathcal{F})=S(\mathcal{G})$ and $S^{0}(\mathcal{F})=S^{0}(\mathcal{G})$.

Corollary 2. Let $0<|T|<\kappa$ and, for each $t \in T$, let a family $\mathcal{F}_{t} \subset$ $\mathcal{P}(X) \backslash\{\emptyset\}$ be given. Assume that:

$1^{0}: \mathcal{F}_{t} \subset S\left(\mathcal{F}_{t}\right)$ for each $t \in T$;

$2^{0}$ : the ideals $S^{0}\left(\mathcal{F}_{t}\right), t \in T$, are pairwise orthogonal;

$3^{0}: \operatorname{add}\left(S^{0}\left(\mathcal{F}_{t}\right)\right) \geq \kappa$ for each $t \in T$.

Then $\bigcap_{t \in T} S\left(\mathcal{F}_{t}\right)=S\left(\bigcup_{t \in T} \mathcal{F}_{t}\right)$ and $\bigcap_{t \in T} S^{0}\left(\mathcal{F}_{t}\right)=S^{0}\left(\bigcup_{t \in T} \mathcal{F}_{t}\right)$.

Proof. Denote $\Sigma^{t}=S\left(\mathcal{F}_{t}\right)$ and $\mathcal{I}^{t}=S^{0}\left(\mathcal{F}_{t}\right)$ for $t \in T$. By $2^{0}$, for any $t_{1}, t_{2} \in T, t_{1} \neq t_{2}$, we can pick a set $A\left(t_{1}, t_{2}\right) \in \mathcal{I}^{t_{1}}$ with $X \backslash A\left(t_{1}, t_{2}\right) \in \mathcal{I}^{t_{2}}$. We may assume that $A\left(t_{2}, t_{1}\right)=X \backslash A\left(t_{1}, t_{2}\right)$. For each $t_{0} \in T$ define

$$
A\left(t_{0}\right)=\bigcup_{t \in T \backslash\left\{t_{0}\right\}} A\left(t_{0}, t\right) .
$$

Then $A\left(t_{0}\right) \in \mathcal{I}^{t_{0}}$, by $3^{0}$ and $|T|<\kappa$. It can easily be checked that $\mathcal{F}_{t} \subset \Sigma^{t}$ (see $1^{0}$ ) implies $\Sigma^{t} \backslash \mathcal{I}^{t} \sim \mathcal{F}_{t}$ for each $t \in T$. (See [2, Proposition 1.1].) Put

$$
\mathcal{F}_{t}^{*}=\left\{A \backslash A(t): A \in \Sigma^{t} \backslash \mathcal{I}^{t}\right\}, \quad t \in T .
$$

Obviously $\mathcal{F}_{t}^{*} \sim \Sigma^{t} \backslash \mathcal{I}^{t}$ and thus $\mathcal{F}_{t}^{*} \sim \mathcal{F}_{t}$ for each $t \in T$. Consider any $t_{1}, t_{2} \in T, t_{1} \neq t_{2}$, and $A_{1} \in \mathcal{F}_{t_{1}}^{*}, A_{2} \in \mathcal{F}_{t_{2}}^{*}$. Then $A_{1} \subset X \backslash A\left(t_{1}\right) \subset$ $X \backslash A\left(t_{1}, t_{2}\right)=A\left(t_{2}, t_{1}\right)$ and $A_{2} \subset X \backslash A\left(t_{2}\right) \subset X \backslash A\left(t_{2}, t_{1}\right)$, so $A_{1} \subset A_{2}$ is 
impossible. Hence by Corollary 1 we obtain $\bigcap_{t \in T} S\left(\mathcal{F}_{t}^{*}\right)=S\left(\bigcup_{t \in T} \mathcal{F}_{t}^{*}\right)$ and $\bigcap_{t \in T} S^{0}\left(\mathcal{F}_{t}^{*}\right)=S^{0}\left(\bigcup_{t \in T} \mathcal{F}_{t}^{*}\right)$. But $\mathcal{F}_{t}^{*} \sim \mathcal{F}_{t}$ for each $t \in T$, and thus also $\bigcup_{t \in T} \mathcal{F}_{t}^{*} \sim \bigcup_{t \in T} \mathcal{F}_{t}$. Hence the assertion follows.

Example 1. In [18] Mycielski introduced a class of $\sigma$-ideals on the Cantor space $2^{\omega}$; we shall call them Mycielski ideals. Further results on that topic were obtained in [3] and [20], [21]. Lemma 1.1 in [3] states that one can find a Mycielski ideal othogonal to each ideal of a given countable family of Mycielski ideals. This easily leads to the family $\left\{\mathcal{M}_{\alpha}: \alpha<\omega_{1}\right\}$ of pairwise orthogonal Mycielski ideals. To apply Corollary 2 we put $\mathcal{F}_{\alpha}=\left\{2^{\omega} \backslash E: E \in\right.$ $\mathcal{M}_{\alpha}$ \}. Then $\mathcal{F}_{\alpha} \subset \mathcal{M}_{\alpha} \cup \mathcal{F}_{\alpha}=S\left(\mathcal{F}_{\alpha}\right)$ and $\mathcal{M}_{\alpha}=S^{0}\left(\mathcal{F}_{\alpha}\right)$ for each $\alpha<\omega_{1}$. (See [2, Proposition 1.5].) Since $\mathcal{M}_{\alpha}$ are $\sigma$-ideals, we have $\operatorname{add}\left(\mathcal{M}_{\alpha}\right) \geq \omega_{1}$ for $\alpha<\omega_{1}$ (in fact $\operatorname{add}\left(\mathcal{M}_{\alpha}\right)=\omega_{1}$, cf. [20]). Hence Corollary 2 applies.

Example 2. Let $X=\mathbb{R}$. Let $\Sigma_{1}$ and $\Sigma_{2}$ stand for the algebra of all Lebesgue measurable sets, and for the algebra of all sets with the Baire property (in $\mathbb{R}$ ). Let $\mathcal{I}_{1}$ and $\mathcal{I}_{2}$ denote the ideal of null sets, and the ideal of meager sets (in $\mathbb{R})$. In [6] it is proved that $\Sigma_{1}=S\left(\mathcal{F}_{1}\right)$ and $\mathcal{I}_{1}=S^{0}\left(\mathcal{F}_{1}\right)$ where $\mathcal{F}_{1}$ consists of perfect sets of positive measure. In [5] it is proved that $\Sigma_{2}=S\left(\mathcal{F}_{2}\right)$ and $\mathcal{I}_{2}=S^{0}\left(\mathcal{F}_{2}\right)$ where $\mathcal{F}_{2}$ consists of sets of the form $U \backslash A$ where $U$ is nonempty open and $A \subset U$ is meager of type $F_{\sigma}$. By Corollary 2 we infer that $\Sigma_{1} \cap \Sigma_{2}=S\left(\mathcal{F}_{1} \cup \mathcal{F}_{2}\right)$ and $\mathcal{I}_{1} \cap \mathcal{I}_{2}=S^{0}\left(\mathcal{F}_{1} \cup \mathcal{F}_{2}\right)$, so the pair $\left(\Sigma_{1} \cap \Sigma_{2}, \mathcal{I}_{1} \cap \mathcal{I}_{2}\right)$ is MB-representable. This result can be also derived from the general theorem by Baldwin [4] who proved that if the pair $(\Sigma, \mathcal{I})$, consisting of an algebra $\Sigma$ and an ideal $\mathcal{I} \subset \Sigma$, possesses the so-called hull property, then $\Sigma=S(\Sigma \backslash \mathcal{I})$ and $\mathcal{I}=S^{0}(\Sigma \backslash \mathcal{I})$. We say that $(\Sigma, \mathcal{I})$ has the hull property if whenever $U \subset X$ there is a $V \in \Sigma$ such that $U \subset V$, and if $W \in \Sigma$ is such that $U \subset W$, then $V \backslash W \in \mathcal{I}$. It is known that each of the pairs $\left(\Sigma_{1}, \mathcal{I}_{1}\right),\left(\Sigma_{2}, \mathcal{I}_{2}\right)$ has the hull property, and the same follows for $\left(\Sigma_{1} \cap \Sigma_{2}, \mathcal{I}_{1} \cap \mathcal{I}_{2}\right)$. Thus $\Sigma_{1} \cap \Sigma_{2}=S\left(\Sigma_{1} \cap \Sigma_{2} \backslash\left(\mathcal{I}_{1} \cap \mathcal{I}_{2}\right)\right)$ and $\mathcal{I}_{1} \cap \mathcal{I}_{2}=$ $S^{0}\left(\Sigma_{1} \cap \Sigma_{2} \backslash\left(\mathcal{I}_{1} \cap \mathcal{I}_{2}\right)\right)$. Finally, observe that $\mathcal{F}_{1} \sim \Sigma_{1} \backslash \mathcal{I}_{1}, \mathcal{F}_{2} \sim \Sigma_{2} \backslash \mathcal{I}_{2}$ and $\mathcal{F}_{1} \cup \mathcal{F}_{2} \sim \Sigma_{1} \cap \Sigma_{2} \backslash\left(\mathcal{I}_{1} \cap \mathcal{I}_{2}\right)$.

Condition $(\star)$ in Theorem 2 is rather restrictive. Let us mention two important algebras of the form $\bigoplus_{Y \in \mathcal{Y}} \Sigma^{Y}$. Because of condition $(\star)$, our Theorem 2 seems useless in these cases. Namely, consider the algebra $\Sigma$ of all sets in $\mathbb{R}$ that are of types $F_{\sigma}$ and $G_{\delta}$ simultaneously. Following [14, $\S 34, \mathrm{VI}]$ we have $\Sigma=\bigoplus_{Y \in \mathcal{Y}} \Sigma^{Y}$ where $\mathcal{Y}$ is the family of all nonempty closed sets in $\mathbb{R}$, and $\Sigma^{Y}$ stands for the algebra of subsets of $Y$ with nowhere dense boundary, relatively to $Y$. It is known that $\Sigma^{Y}=S_{Y}\left(\mathcal{F}_{Y}\right)$ where $\mathcal{F}_{Y}$ consists of all nonempty sets open in the topology of $Y$. A trouble with condition $(\star)$ appears in the following situation. Let $Y_{1}, Y_{2}$ be nonempty perfect sets 
where $Y_{1} \subset Y_{2}$ and $Y_{1}$ is nowhere dense in $Y_{2}$. Then for each $A_{1} \in \mathcal{F}_{Y_{1}}$ we have $A_{1} \notin \mathcal{F}_{Y_{2}}$.

Let us consider another example. Let $\mathcal{Y}$ be as above and now let $\Sigma^{Y}$ stand for the algebra of subsets of $Y \in \mathcal{Y}$, with the Baire property, relatively to $Y$. Thus the sets of the algebra $\Sigma=\bigoplus_{Y \in \mathcal{Y}} \Sigma^{Y}$ are called sets with the Baire property in the restricted sense. (See $[14, \S 11, \mathrm{VI}]$.) Theorem 2 again seems unapplicable.

We leave open the question whether these two algebras are MBrepresentable. The problem concerning sets with the Baire property in the restricted sense has been suggested to the first author by P. Reardon.

\section{MB-representations of algebras $\Delta_{\alpha}^{0}$}

It was shown in [1] that, under GCH (more precisely, under $2^{\omega}=\omega_{1}$ and $2^{\omega_{1}}=\omega_{2}$ ), the algebra of all Borel sets in $\mathbb{R}$ is MB-representable. We will observe that the same method leads to the analogous result for the algebras of ambiguous Borel sets of classes $\alpha \geq 3$. However, this does not work in the case $\alpha=2$.

Recall necessary definitions from [1]. Let $\mathcal{A} \subset \mathcal{P}(X)$ and $\mathcal{I} \subset \mathcal{A}$ be an algebra and an ideal. We say that $\mathcal{A}$ is inner (outer) $M B$-representable if there is an $\mathcal{F} \subset \mathcal{P}(X)$ such that $\mathcal{A}=S(\mathcal{F})$ and $\mathcal{F} \subset \mathcal{A}(\mathcal{F} \cap \mathcal{A}=\emptyset)$. We say that $\mathcal{A}$ is strongly outer $M B$-representable if for each family $\mathcal{C} \subset \mathcal{P}(X)$ with $\mathcal{A} \subset \mathcal{C}$ and $|\mathcal{C}|=|\mathcal{A}|$ there is an $\mathcal{F} \subset \mathcal{P}(X) \backslash \mathcal{C}$ such that $\mathcal{A}=S(\mathcal{F})$. If moreover, $\mathcal{I}=S^{0}(\mathcal{F})$, we say that the pair $(\mathcal{A}, \mathcal{I})$ is (respectively) inner, or outer, or strongly outer MB-representable. We shall use, in the role of $\mathcal{I}$, the ideal

$$
H(\mathcal{A})=\{A \subset X:(\forall B \subset A) B \in \mathcal{A}\}
$$

of sets which hereditarily belong to $\mathcal{A}$.

Let us quote two theorems from [1].

Theorem 3. Let $|X|=\kappa \geq \omega$ and let $\mathcal{A} \subset \mathcal{P}(X)$ be an algebra such that $H(\mathcal{A}) \subset[X]^{<\kappa}, \mathcal{A} \cap[X]^{<\kappa} \subset H(\mathcal{A})$ and $S\left(\mathcal{A} \backslash[X]^{<\kappa}\right) \backslash \mathcal{A} \neq \emptyset$. Then $\mathcal{A}$ is not inner $M B$-representable.

Theorem 4. Let $|X|=\kappa \geq \omega$ and let $\mathcal{A} \subset \mathcal{P}(X)$ be an algebra such that $[X]^{<\kappa} \subset \mathcal{A}$. If $2^{\kappa}=\kappa^{+}$and $|\mathcal{A}|<2^{\kappa}$ then the pair $(\mathcal{A}, H(\mathcal{A}))$ is strongly outer $M B$-representable.

Fix an uncountable Polish space $X$. Thus $|X|=\mathfrak{c}$ where $\mathfrak{c}=2^{\omega}$ is the cardinality of continuum. Consider $\Delta_{\alpha}^{0}, \alpha<\omega_{1}$, the algebra of ambiguous Borel sets of class $\alpha$ in $X$, i.e. $\Delta_{2}^{0}=F_{\sigma} \cap G_{\delta}, \Delta_{3}^{0}=F_{\sigma \delta} \cap G_{\delta \sigma}$, etc. (See 
[11].) The algebra $\Delta_{\omega_{1}}^{0}=\bigcup_{\alpha<\omega_{1}} \Delta_{\alpha}^{0}$ consists of all Borel sets in $X$. The family of $G_{\delta}$ sets in $X$ is written as $\Pi_{2}^{0}$.

Lemma 1. $H\left(\Delta_{2}^{0}\right)=[X] \leq \omega \cap \Pi_{2}^{0}$.

Proof. To show " $\subset$ " suppose that $A \in H\left(\Delta_{2}^{0}\right)$ is uncountable. Pick a perfect subset of $A$ and its subset which is not in $\Delta_{2}^{0}$. (See $[11,13.6,22.4]$.) Contradiction.

To show " $\supset$ " consider an $A \in[X] \leq \omega \cap \Pi_{2}^{0}$. Let $B \subset A$. Thus $X \backslash B=$ $(X \backslash A) \cup(A \backslash B)$ and so, $B$ is of type $G_{\delta}$. Hence $B \in[X]^{\leq \omega} \cap \Pi_{2}^{0} \subset \Delta_{2}^{0}$. Consequently $A \in H\left(\Delta_{2}^{0}\right)$.

Lemma 2. $H\left(\Delta_{\alpha}^{0}\right)=[X] \leq \omega$ for each $\alpha, 3 \leq \alpha \leq \omega_{1}$.

Proof. The argument for " $\subset$ " is similar to that in the proof of Lemma 1. Inclusion " $\supset$ " follows from $[X] \leq \omega \subset \Delta_{3}^{0} \subset \Delta_{\alpha}^{0}$.

As an application of Theorems 3 and 4 we obtain the following

Theorem 5. In an uncountable Polish space $X$, we have:

(I) algebras $\Delta_{\alpha}^{0}, 2 \leq \alpha \leq \omega_{1}$, are not inner MB-representable;

(II) if $2^{\omega}=\omega_{1}$ and $2^{\omega_{1}}=\omega_{2}$, then the pairs $\left(\Delta_{\alpha}^{0},[X]^{\omega}\right), 3 \leq \alpha \leq \omega_{1}$, are strongly outer MB-representable.

Proof. (I) (Cf. [1, Corollary 14].) Fix $\alpha, 2 \leq \alpha \leq \omega_{1}$, and put $\mathcal{A}=\Delta_{\alpha}^{0}$. From Lemmas 1 and 2 we infer that $H(\mathcal{A}) \subset[X]^{<\mathfrak{c}}$ and $\mathcal{A} \cap[X]^{<\mathfrak{c}} \subset$ $H(\mathcal{A})$. Observe that $\mathcal{A} \backslash[X]^{<\mathfrak{c}}$ and the family of all perfect sets in $X$ are mutually coinitial. Hence $S\left(\mathcal{A} \backslash[X]^{<\mathfrak{c}}\right)$ is equal to the algebra of Marczewski measurable sets. Since there is a non-Borel Marczewski measurable set [15], we may use Theorem 3 .

(II) (Cf. [1, Corollary 5].) We have $|X|=\mathfrak{c}=\omega_{1}$, and $[X]^{<\omega_{1}}=[X]^{\leq \omega}=$ $H\left(\Delta_{\alpha}^{0}\right) \subset \Delta_{\alpha}^{0}$ by Lemma 2. Also $\left|\Delta_{\alpha}^{0}\right|=\mathfrak{c}=\omega_{1}<\omega_{2}=2^{\omega_{1}}$. Then apply Theorem 4 .

Remark 2. In the case $\alpha=2$ we cannot repeat the argument for Theorem 5 (II) since $[X]^{\omega} \subset \Delta_{2}^{0}$ is false. Indeed, each set from $[X]^{\omega} \backslash \Pi_{2}^{0}$ is not in $\Delta_{2}^{0}$.

Finally, let us show that Theorem 4 can be applied to an algebra on a set of cardinality $2^{\lambda}$ where a cardinal $\lambda \geq \omega$ is arbitrarily large. 
Example 3. Let $\lambda \geq \omega$ be a cardinal and consider the Cantor cube $X=$ $\{0,1\}^{\lambda}$. The basis for the product topology $\tau$ on $X$ is of size $\left|[\lambda]^{<\omega}\right|=\lambda$ which easily implies that $|\tau|=2^{\lambda}$. Hence $|X|=|\tau|=2^{\lambda}$ and we denote $2^{\lambda}=\kappa$. An algebra $\mathcal{A} \subset \mathcal{P}(X)$ will be called $\lambda^{+}$-additive if $\bigcup_{\nu<\lambda} A_{\nu} \in \mathcal{A}$ for any function $\nu \mapsto A_{\nu} \in \mathcal{A}, \nu<\lambda$. Now, let $\mathcal{A}$ stand for the smallest $\lambda^{+}$additive algebra containing $\tau$. We can classify sets in $\mathcal{A}$ analogously as Borel sets in a Polish space, considering the classes analogous to $\Sigma_{\alpha}^{0}, \Pi_{\alpha}^{0}$ (cf. [11], [7]) but now $\alpha<\lambda^{+}$. Each of this class is of size $\kappa$ since $\kappa^{\lambda}=\left(2^{\lambda}\right)^{\lambda}=\kappa$. Hence we conclude that $|\mathcal{A}| \leq \lambda^{+} \kappa=\kappa$. Assume $2^{\lambda}=\lambda^{+}$(that is $\kappa=\lambda^{+}$) and $2^{\kappa}=\kappa^{+}$, which is a part of GCH. Thus $[X]^{<\kappa}=[X]^{\leq \lambda} \subset \mathcal{A}$ and $|\mathcal{A}|=\kappa<2^{\kappa}$. Consequently, Theorem 4 applies.

\section{MB-representations of clopen sets}

A basic question concerning MB-representations was whether every algebra of sets is MB-representable. Now, the answer is known. One of the theorems in [1] gives the negative answer under GCH: if $2^{\kappa}=\kappa^{+}$and $|X|=\kappa \geq \omega$ then there is a non-MB-representable algebra on $X$. In December 2002, P. Koszmider [13] found a non-MB-representable algebra $\mathcal{A} \subset \mathcal{P}(\omega)$ in $\mathrm{ZFC}$.

A related question is whether every algebra of sets is isomorphic to an MB-representable algebra where an isomorphism is meant in the Boolean theoretical sense. Suprisingly, Koszmider [13] answered it in the affirmative. A natural idea to solve this problem is to use the classical Stone representation theorem which states that every Boolean algebra (in particular, every algebra of sets) is isomorphic to the algebra $\operatorname{Clop}(X)$ of clopen subsets of some zero-dimensional compact Hausdorff space $X$. (See [12].) Koszmider [13] proved that this last algebra is isomorphic to an MB-representable algebra of sets. Independently of this result one can pose the following topological problem: describe all zero-dimensional compact Hausdorff spaces $X$ for which $\operatorname{Clop}(X)$ is MB-representable. We do not solve it in this paper. We only give some conditions on a topological space $X$ under which the algebra $\operatorname{Clop}(X)$ is MB-representable.

Let $\lambda \geq 2$ be a cardinal. A topological space is called $\lambda$-resolvable if there is a disjoint family of cardinality $\lambda$, of dense subsets of $X$. (See [8].) Clearly, each $\lambda$-resolvable space is dense-in-itself. Now, let $\lambda$ be infinite. For $\Gamma \subset \lambda$ put $(+1) \Gamma=\Gamma$ and $(-1) \Gamma=\Gamma^{c}(=\lambda \backslash \Gamma)$. A family $\mathcal{F} \subset \mathcal{P}(\lambda)$ is called independent if, whenever $\Gamma_{0}, \ldots, \Gamma_{n}$ is a finite sequence of distinct elements from $\mathcal{F}$ and $\varepsilon_{0}, \ldots, \varepsilon_{n}$ is a sequence of numbers $-1,+1$, then $\bigcap_{k=0}^{n} \varepsilon_{k} \Gamma_{k} \neq \emptyset$. The theorem of Fichtenholz, Kantorovitch and Hausdorff states that for each cardinal $\lambda \geq \omega$ there is an independent family $\mathcal{F} \subset \mathcal{P}(\lambda)$ of cardinality $2^{\lambda}$. (See [16].) We call it Theorem FKH. 
Theorem 6. Let $X$, with $|X|=\kappa \geq \omega$, be a dense-in-itself a $\lambda$-resolvable topological space where $2^{\lambda} \geq \kappa$. Then the algebra $\operatorname{Clop}(X)$ is $M B$ representable.

Proof. We will mimic some ideas contained in [2, Theorem 2.1] which are due to S. Wroński. The trick with an independent family, suggested us by P. Koszmider, has strenghtened the former version of our theorem.

By Theorem FKH there is an independent family $\mathcal{T} \subset \mathcal{P}(\lambda)$ of size $2^{\lambda}$. Since $\kappa \leq 2^{\lambda}$, any subfamily of size $\kappa$ is also independent. So, assume that $|\mathcal{T}|=\kappa$. Because $|X|=\kappa$, we may put $\mathcal{T}=\left\{T_{x}: x \in X\right\}$. Since $X$ is $\lambda$-resolvable, there is a disjoint family $\left\{D_{\alpha}: \alpha<\lambda\right\}$ of dense subsets of $X$. We may assume that $\bigcup_{\alpha<\lambda} D_{\alpha}=X$. Define

$$
F(x)=\bigcup_{\alpha \in T_{x}} D_{\alpha} \backslash\{x\} \text { for } x \in X .
$$

Obviously, the sets $F(x), x \in X$, are dense.

Claim 1. For each finite set $\left\{x_{0}, \ldots, x_{n}\right\} \subset X$, the set $X \backslash \bigcup_{k=0}^{n} F\left(x_{k}\right)$ is dense.

Indeed, by the definition of $F\left(x_{k}\right)$ and the disjointness of sets $D_{\alpha}$ we have

$$
X \backslash \bigcup_{k=0}^{n} F\left(x_{k}\right) \supset \bigcap_{k=0}^{n} \bigcup_{\alpha \in T_{x_{k}}^{c}} D_{\alpha} \supset \bigcup_{\alpha \in T_{x_{0}}^{c} \cap \cdots \cap T_{x_{n}}^{c}} D_{\alpha} .
$$

Since $\mathcal{T}$ is independent, $T_{x_{0}}^{c} \cap \cdots \cap T_{x_{n}}^{c} \neq \emptyset$. Thus $X \backslash \bigcup_{k=0}^{n} F\left(x_{k}\right)$ contains at least one dense set $D_{\alpha}$ which ends the proof of Claim 1.

Now, define $\mathcal{F}=\{U \backslash F(x): x \in U$ and $U$ is open $\}$.

At first we shall prove that $\operatorname{Clop}(X) \subset S(\mathcal{F})$. Let $V \in \operatorname{Clop}(X)$ and consider a $U \backslash F(x) \in \mathcal{F}$. There are two cases:

$1^{0}: x \in V$. Then $x \in U \cap V$. Put $W=U \cap V$. Hence $W \backslash F(x) \subset$ $(U \backslash F(x)) \cap V$ and $W \backslash F(x) \in \mathcal{F}$.

$2^{0}: x \notin V$. Then $x \in U \backslash V$ and from $V \in \operatorname{Clop}(X)$ it follows that the set $W=U \backslash V$ is open. Hence $W \backslash F(x) \subset(U \backslash F(x)) \backslash V$ and $W \backslash F(x) \in \mathcal{F}$.

To prove $S(\mathcal{F}) \subset \operatorname{Clop}(X)$ we need the following:

Claim 2. If $V \backslash F(y)$ and $U \backslash F(x)$ are in $\mathcal{F}$ and $V \backslash F(y) \subset U \backslash F(x)$, then $x=y$.

Indeed, suppose that $x \neq y$. Then

$$
\begin{aligned}
V & =(V \backslash F(y)) \cup(V \cap F(y)) \subset(X \backslash F(x)) \cup F(y) \\
& \subset\{x\} \cup \bigcup_{\alpha \in T_{x}^{c}} D_{\alpha} \cup \bigcup_{\alpha \in T_{y}} D_{\alpha} \subset\{x\} \cup\left(X \backslash \bigcup_{\alpha \in T_{x} \cap T_{y}^{c}} D_{\alpha}\right) .
\end{aligned}
$$


By the independence of $\mathcal{T}$, the set $T_{x} \cap T_{y}^{c}$ is nonempty, so there is a dense set $D_{\alpha}$, for some $\alpha \in T_{x} \cap T_{y}^{c}$, disjoint from the nonempty open set $V \backslash\{x\}$. Contradiction.

Now, let $A \in S(\mathcal{F})$. We want to show that $A$ is open. Since we may replace $A$ by $X \backslash A$, this will end the proof. Suppose that $A$ is not open. Thus there exists an $x \in A \backslash \operatorname{int} A$. Clearly $X \backslash F(x) \in \mathcal{F}$. Since $A \in S(\mathcal{F})$ there is $U \backslash F(y) \in \mathcal{F}$ such that either

$$
U \backslash F(y) \subset(X \backslash F(x)) \cap A
$$

or $U \backslash F(y) \subset(X \backslash F(x)) \backslash A$. By Claim 2 we have $x=y$. Since $x=y \in$ $(U \backslash F(y)) \cap A$, condition (1) holds. From $x \in U$ and $x \notin \operatorname{int} A$ it follows that $U \backslash A \neq \emptyset$. Pick a $z \in U \backslash A$. Thus $U \backslash F(z) \in \mathcal{F}$ and from $A \in S(\mathcal{F})$ it follows that there is $V \backslash F(t) \in \mathcal{F}$ such that either $V \backslash F(t) \subset(U \backslash F(z)) \cap A$ or

$$
V \backslash F(t) \subset(U \backslash F(z)) \backslash A .
$$

Using Claim 2 again we infer that $z=t$. Since $z=t \in(V \backslash F(t)) \backslash A$, condition (2) holds. By Claim 1 we have $V \backslash(F(x) \cup F(z)) \neq \emptyset$. On the other hand,

$$
V \backslash(F(x) \cup F(z)) \subset U \backslash F(x)=U \backslash F(y) \subset A, \text { by }(1)
$$

and

$$
V \backslash(F(x) \cup F(z)) \subset V \backslash F(z)=V \backslash F(t) \subset X \backslash A, \text { by (2). }
$$

Contradiction.

Example 4. Let $\eta \geq \omega$ be a cardinal and put $\lambda=2^{\eta}, \kappa=2^{\lambda}$. Consider Cantor cubes $X_{1}=\{0,1\}^{\eta}, X_{2}=\{0,1\}^{\lambda}$. Then $\Delta\left(X_{1}\right)=\lambda, \Delta\left(X_{2}\right)=\kappa$. Let $X$ be a topological sum of $X_{1}$ and $X_{2}$. (See [9].) Thus $|X|=\lambda+\kappa=\kappa$ and $\Delta(X)=\lambda$. The space $X$ is compact and dense-in-itself. So by [8, Theorem 3.7] the space $X$ is $\lambda$-resolvable. Thus we may apply Theorem 6 with $2^{\lambda}=\kappa$. Hence $\operatorname{Clop}(X)$ is $\mathrm{MB}$-representable. Note that $\operatorname{Clop}(X)$ is nontrivial since $X$ is zero-dimensional as a sum of zero-dimensional spaces $X_{1}$ and $X_{2}$. (See [9].) If we consider space $X_{2}$ instead of $X$, we get a simple example where Theorem 6 applies (thus $\left|X_{2}\right|=\kappa$ and $X_{2}$ is $\kappa$-resolvable) but in this case we do not use the whole power of our result.

It is an easy observation that, for a discrete topological space $X$, we have $\operatorname{Clop}(X)=\mathcal{P}(X)=S(\mathcal{P}(X) \backslash\{\emptyset\})$. This mixed with Theorem 6 produces the following 
Corollary 3. Let $X$ be a topological space which is a sum of (pairwise disjoint, clopen) subspaces $X_{t}, t \in T$, such that each $X_{t}$ is either discrete, or $\left|X_{t}\right|=\kappa_{t} \geq \omega$ and $X_{t}$ is $\lambda_{t}$-resolvable with $2^{\lambda_{t}} \geq \kappa_{t}$. Then $\operatorname{Clop}(X)$ is MB-representable.

Proof. Let $\Sigma=\operatorname{Clop}(X)$ and $\Sigma^{t}=\operatorname{Clop}\left(X_{t}\right)$ for $t \in T$. Then $\Sigma^{t}$ are MBrepresentable by Theorem 6 and the above observation. The rest follows from Theorem 2.

Acknowledgements. We would like to thank Piotr Koszmider for his advice that helped us to improve Theorem 6 .

\section{References}

[1] Balcerzak, M., Bartoszewicz, A., Ciesielski, K., On Marczewski-Burstin representations of certain algebras of sets, Real Anal. Exchange 26 (2000/2001), 581-592.

[2] Balcerzak, M., Bartoszewicz, A., Rzepecka, J., Wroński, S., Marczewski fields and ideals, Real Anal. Exchange 26 (2000/2001), 703-715.

[3] Balcerzak, M., Rosłanowski, A., On Mycielski ideals, Proc. Amer. Math. Soc. 110 (1990), 243-250.

[4] Baldwin, S., The Marczewski hull property and complete Boolean algebras, Real Anal. Exchange, (to appear).

[5] Brown, J. B., Elalaoui-Talibi, H., Marczewski-Burstin-like characterizations of $\sigma$ algebras, ideals, and measurable functions, Colloq. Math. 82 (1999), 277-286.

[6] Burstin, C., Eigenschaften messbaren und nichtmessbaren Mengen, Wien Ber. 123 (1914), 1525-1551.

[7] Ciesielski, K.,Set Theory for the Working Mathematician, London Math. Soc. Stud. Texts 39, Cambridge Univ. Press, Cambridge, 1997.

[8] Comfort, W. W., Garcia-Ferreira, S., Resolvability: a selective survey and some new results, Topology Appl. 74 (1996), 149-167.

[9] Engelking, R., General Topology, PWN, Warsaw 1977.

[10] Hewitt, E., A problem of set-theoretic topology, Duke Math. J. 10 (1943), 309-333.

[11] Kechris, A. S., Classical Descriptive Set Theory, Springer, New York, 1994.

[12] Koppelberg, S., Handbook of Boolean Algebras, Vol. 1, Elsevier, Amsterdam, 1989.

[13] Koszmider, P., unpublished notes.

[14] Kuratowski, K., Topology, Vol.1, Academic Press, New York, 1966.

[15] Miller, A. W., Special subsets of the real line, in: "Handbook of Set Theoretic Topology", K. Kunen and J. E. Vaughan, eds., Elsevier, Amseterdam, 1984.

[16] Monk, J. D., Appendix on set theory, in: "Handbook of Boolean Algebras", Vol. 3, Elsevier, Amsterdam, 1989, 1215-1233.

[17] Morgan II, J. C., Point Set Theory, Marcel Dekker, New York, 1990.

[18] Mycielski, J., Some new ideals of sets on the real line, Colloq. Math. 20 (1969), 71-76.

[19] Pawlikowski, J., Parametrized Ellentuck theorem, Topology Appl. 37 (1990), 65-73.

[20] Rosłanowski, A., On game ideals, Colloq. Math. 59 (1990), 159-168. 
[21] Rosłanowski, A., Mycielski ideals generated by uncountable systems, Colloq. Math. 66 (1994), 187-200.

[22] Szpilrajn (Marczewski), E., Sur une classe de fonctions de M. Sierpiński et la classe correspondante d'ensembles, Fund. Math. 24 (1935), 17-34.

MAREK BALCERZAK

InSTITUTE OF Mathematics

ŁÓDŹ TECHNICAL UNIVERSITY

Al. Politechniki 11

90-924 ŁóDŹ, POLAND

AND

FACUlty of Mathematics

UNIVERSITY OF ŁÓDŹ

BANACHA 22

90-238 ŁÓDŹ, POLAND

MBALCE@UNI.LODZ.PL
JoANNA RZEPECKA

INSTITUTE OF MATHEMATICS

ŁÓDŹ TECHNICAL UNIVERSITY

Al. Politechniki 11

90-924 ŁÓDŹ, POLAND 